ISSN 2078-3744. Вісник Львів. ун-ту. Серія мех.-мат. 2019. Випуск 88. С. 83-97

Visnyk of the Lviv Univ. Series Mech. Math. 2019. Issue 88. P. 83-97

http://publications.lnu.edu.ua/bulletins/index.php/mmf

doi: http://dx.doi.org/10.30970/vmm.2019.88.083-097

УДК 517.537 .72

\title{
ON HADAMARD COMPOSITIONS OF ENTIRE DIRICHLET SERIES AND DIRICHLET SERIES ABSOLUTELY CONVERGING IN HALF-PLANE
}

\author{
Oksana MULYAVA $^{1}$, Myroslav SHEREMETA $^{2}$ \\ ${ }^{1}$ Kyiv National University of Food Technologies \\ Volodymyrska Str., 68, 01033, Kyiv \\ e-mail: oksana.m@bigmir.net \\ ${ }^{2}$ Ivan Franko National University of Lviv, \\ Universytetska Str., 1, 79000, Lviv \\ e-mail: m.m.sheremeta@gmail.com
}

For an entire Dirichlet series $F(s)=\sum_{k=0}^{\infty} f_{k} \exp \left\{s \lambda_{k}\right\}$ and a Dirichlet series $G(s)=\sum_{k=0}^{\infty} g_{k} \exp \left\{s \lambda_{k}\right\}$ with finite abscissa of the absolute convergence the Dirichlet series $(F * G)(s)=\sum_{k=0}^{\infty} f_{k} g_{k} \exp \left\{s \lambda_{k}\right\}$ is called the Hadamard composition. In terms of generalized orders the growth of this composition and their derivatives is investigated. A relation between the behavior of the maximal terms of the Hadamard composition of the derivatives and of the derivative of the Hadamard composition is established.

Key words: Dirichlet series, Hadamard composition, generalized order, maximal term.

\section{INTRODUCTION}

For power series $f(z)=\sum_{k=0}^{\infty} f_{k} z^{k}$ and $g(z)=\sum_{k=0}^{\infty} g_{k} z^{k}$ with the convergence radii $R[f]$ and $R[g]$ the series $(f * g)(z)=\sum_{k=0}^{\infty} f_{k} g_{k} z^{k}$ is called the Hadamard composition. It is well known $[1,2]$ that $R[f * g] \geq R[f] R[g]$. Properties of this composition obtained by

2010 Mathematics Subject Classification: 30B50, 30D15

(C) Mulyava, O., Sheremeta, M., 2019 
J. Hadamard find applications [2, 3] in the theory of analytic continuation of the functions represented by power series. We remark also that singular points of the Hadamard composition are investigated in the article [4].

For $0 \leq r<R[f]$ let $\mu_{f}(r)=\max \left\{\left|f_{k}\right| r^{k}: k \geq 0\right\}$ be the maximal term of the power expansion of $f$. Studying [5,6] a connection between the growth of maximal terms of a derivative of the Hadamard's composition of two entire functions $f$ and $g$ and the Hadamard composition of their derivatives M. Sen [6], in particular proved, that if the function $(f * g)$ has order $\varrho$ and lower order $\lambda$ then for every $\varepsilon>0$ and all $r \geq r_{0}(\varepsilon)$

$$
r^{(n+2) \lambda-1-\varepsilon} \leq \frac{\mu_{f^{(n+1)} * g^{(n+1)}}(r)}{\mu_{(f * g)^{(n)}}(r)} \leq r^{(n+2) \varrho-1+\varepsilon} .
$$

Since Dirichlet series with positive increasing to $+\infty$ exponents are direct generalizations of power series, a problem becomes natural on similar results for a Hadamard composition of such series.

So, let $\Lambda=\left(\lambda_{k}\right)$ be an increasing to $+\infty$ sequence of nonnegative numbers $\left(\lambda_{0}=0\right)$, and $S(\Lambda, A)$ be a class of Dirichlet series

$$
F(s)=\sum_{k=0}^{\infty} f_{k} \exp \left\{s \lambda_{k}\right\}, \quad s=\sigma+i t
$$

with the exponents $\Lambda$ and the abscissa of absolute convergence $\sigma_{a}[F]=A$. If $F \in\left(\Lambda, A_{1}\right)$ and $G(s)=\sum_{k=0}^{\infty} g_{k} \exp \left\{s \lambda_{k}\right\} \in\left(\Lambda, A_{2}\right)$ the Dirichlet series

$$
(F * G)(s)=\sum_{k=0}^{\infty} f_{k} g_{k} \exp \left\{s \lambda_{k}\right\}
$$

is called [7] the Hadamard composition of $F$ and $G$.

For a Dirichlet series (1) with $\sigma_{a}[F]=A[F]=A>-\infty$ and $\sigma<A$ we put $M(\sigma, F)=\sup \{|F(\sigma+i t)|: t \in \mathbb{R}\}$, and let $\mu(\sigma, F)=\max \left\{\left|f_{k}\right| \exp \left\{\sigma \lambda_{k}\right\}: k \geq 0\right\}$ be the maximal term, $\nu(\sigma, F)=\max \left\{k:\left|f_{k}\right| \exp \left\{\sigma \lambda_{k}\right\}=\mu(\sigma, F)\right\}$ be the central index and $\Lambda(\sigma, F)=\lambda_{\nu(\sigma, F)}$. The following result is proved in [7].

Proposition 1. Let $n \in \mathbb{Z}_{+}, m \in \mathbb{N}$ and $m>n$. If $\sigma_{a}[F]=\sigma_{a}[G]=+\infty$ and $\ln k=$ $=o\left(\lambda_{k} \ln \lambda_{k}\right)$ as $k \rightarrow \infty$ then

$$
\varlimsup_{\sigma \rightarrow+\infty} \frac{1}{\sigma} \ln \frac{\mu\left(\sigma,(F * G)^{(m)}\right)}{\mu\left(\sigma,(F * G)^{(n)}\right)}=(m-n) \varrho_{R}[f * G]
$$

and (if $\left.\varrho_{R}[f * G]<+\infty\right)$

$$
\varliminf_{\sigma \rightarrow+\infty} \frac{1}{\sigma} \ln \frac{\mu\left(\sigma,(F * G)^{(m)}\right)}{\mu\left(\sigma,(F * G)^{(n)}\right)}=(m-n) \lambda_{R}[f * G],
$$

where $\varrho_{R}[f]$ and $\lambda_{R}[f]$ are respectively the $R$-order and the lower $R$-order of entire Dirichlet series (1). If $\sigma_{a}[F]=\sigma_{a}[G]=0$ and $\ln k=o\left(\lambda_{k} / \ln \lambda_{k}\right)$ as $k \rightarrow \infty$ then

$$
\varlimsup_{\sigma \uparrow 0}|\sigma| \ln \frac{\mu\left(\sigma,(F * G)^{(m)}\right)}{\mu\left(\sigma,(F * G)^{(n)}\right)}=(m-n) \varrho^{(0)}[f * G]
$$


and

$$
\varliminf_{\sigma \uparrow 0}|\sigma| \ln \frac{\mu\left(\sigma,(F * G)^{(m)}\right)}{\mu\left(\sigma,(F * G)^{(n)}\right)}=(m-n) \lambda^{(0)}[f * G],
$$

where $\varrho^{(0)}[f]$ and $\lambda^{(0)}[f]$ are respectively the order and the lower order of Dirichlet series (1) with $\sigma_{a}[F]=0$.

Here we will consider the case, when $\sigma_{a}[F]=+\infty$ and $\sigma_{a}[G] \in(-\infty+\infty)$.

\section{ConVERgenCE AND GRoWth}

We put

$$
A[F]=\varliminf_{k \rightarrow+\infty} \frac{1}{\lambda_{k}} \ln \frac{1}{\left|f_{k}\right|}, \quad \bar{A}[F]=\varlimsup_{k \rightarrow+\infty} \frac{1}{\lambda_{k}} \ln \frac{1}{\left|f_{k}\right|} .
$$

It is known ([8],[9]) that $\sigma_{a}[F] \leq A[F]$ and if $\ln k=o\left(\lambda_{k}\right)$ as $k \rightarrow \infty$ then $\sigma_{a}[F]=A[F]$. It is easy to see that if $A[F]>-\infty$ and $A[G]>-\infty$ then $A[F * G] \geq A[F]+A[G]$. Therefore, if $\sigma_{a}[F]=+\infty$ and $A[G]>-\infty$ then $A[F * G]=+\infty$.

We remark also [7] that if $\sigma_{a}[F]=+\infty$ and $\sigma_{a}[G]>-\infty$ then

$$
\sigma_{a}[F * G] \geq \sigma_{a}[F]+\sigma_{a}[G]=+\infty .
$$

Further, we will also assume that $\sigma_{a}[G]=A[G]$.

In $[7]$ it is proved that

$$
\sigma_{a}[F * G]=\sigma_{a}\left[(F * G)^{(n)}\right]=\sigma_{a}\left[F^{(n)} * G^{(n)}\right]
$$

for every $n \in \mathbb{N}$, whence we get the following statement.

Proposition 2. If $\sigma_{a}[F]=+\infty$ and $\sigma_{a}[G]>-\infty$ then

$$
\sigma_{a}[F * G]=\sigma_{a}\left[(F * G)^{(n)}\right]=\sigma_{a}\left[F^{(n)} * G^{(n)}\right]=\sigma_{a}[F]=+\infty
$$

for every $n \in \mathbb{N}$.

By $L$ we denote the class of non-negative continuous on $(-\infty,+\infty)$ functions $\alpha$ such that $\alpha(x)=\alpha\left(x_{0}\right) \geq 0$ for $x \leq x_{0}$ and $\alpha(x) \uparrow+\infty$ as $x_{0} \leq x \rightarrow+\infty$. We say that $\alpha \in L^{0}$, if $\alpha \in L$ and $\alpha((1+o(1)) x)=(1+o(1)) \alpha(x)$ as $x \rightarrow+\infty$. Finally, $\alpha \in L_{s i}$, if $\alpha \in L$ and $\alpha(c x)=(1+o(1)) \alpha(x)$ as $x \rightarrow+\infty$ for each $c \in(0,+\infty)$, i.e. $\alpha$ is a slowly increasing function. Clearly, $L_{s i} \subset L^{0}$.

If $\alpha \in L, \beta \in L$ and $F \in(\Lambda,+\infty)$ then the quantities

$$
\varrho_{\alpha, \beta}[F]:=\varlimsup_{\sigma \rightarrow+\infty} \frac{\alpha(\ln M(\sigma, F))}{\beta(\sigma)}, \quad \lambda_{\alpha, \beta}[F]:=\lim _{\sigma \rightarrow+\infty} \frac{\alpha(\ln M(\sigma, F))}{\beta(\sigma)}
$$

are called the generalized $(\alpha, \beta)$-order and the generalized lower $(\alpha, \beta)$-order of $F$. If in (3) we substitute $\ln \mu(\sigma, F)$ instead of $\ln M(\sigma, F)$ then we obtain quantities, which we denote by $\varrho_{\alpha, \beta}[\ln \mu, F]$ and $\lambda_{\alpha, \beta}[\ln \mu, F]$ respectively. Substituting $\Lambda(\sigma, F)$ instead of $\ln M(\sigma, F)$ by analogy we define $\varrho_{\alpha, \beta}[\Lambda, F]$ and $\lambda_{\alpha, \beta}[\Lambda, F]$. The following lemma is true $[9,10]$. 
Lemma 1. Let $\alpha \in L_{\text {si }}, \beta \in L^{0}$ and $\frac{d \beta^{-1}(c \alpha(x))}{d \ln x}=O(1)$ as $x \rightarrow+\infty$ for each $c \in(0,+\infty)$ and $F \in(\Lambda,+\infty)$. If for each $c \in(0,+\infty)$

$$
\ln k=o\left(\lambda_{k} \beta^{-1}\left(c \alpha\left(\lambda_{k}\right)\right)\right), \quad k \rightarrow \infty,
$$

then

$$
\varrho_{\alpha, \beta}[F]=\varrho_{\alpha, \beta}[\ln \mu, F]=\varlimsup_{k \rightarrow \infty} \frac{\alpha\left(\lambda_{k}\right)}{\beta\left(\frac{1}{\lambda_{k}} \ln \frac{1}{\left|f_{k}\right|}\right)}
$$

If, moreover, $\alpha\left(\lambda_{k+1}\right) \sim \alpha\left(\lambda_{k}\right)$ and $\kappa_{k}[F]:=\frac{\ln \left|f_{k}\right|-\ln \left|f_{k+1}\right|}{\lambda_{k+1}-\lambda_{k}} \nearrow+\infty$ as $k_{0} \leq k \rightarrow \infty$ then

$$
\varrho_{\alpha, \beta}[F]=\varrho_{\alpha, \beta}[\ln \mu, F]=\varliminf_{k \rightarrow \infty} \frac{\alpha\left(\lambda_{k}\right)}{\beta\left(\frac{1}{\lambda_{k}} \ln \frac{1}{\left|f_{k}\right|}\right)} .
$$

We need also the following lemmas.

Lemma 2. If $F \in(\Lambda,+\infty), \alpha\left(e^{x}\right) \in L_{s i}, \beta \in L^{0}$ and $\alpha(x)=o(\beta(x))$ as $x \rightarrow+\infty$ then $\varrho_{\alpha, \beta}[\ln \mu, F]=\varrho_{\alpha, \beta}[\Lambda, F]$ and $\lambda_{\alpha, \beta}[\ln \mu, F]=\lambda_{\alpha, \beta}[\Lambda, F]$.

Proof. We use the equality (see [8], [9])

$$
\ln \mu(\sigma, F)-\ln \mu(0, F)=\int_{0}^{\sigma} \Lambda(x) d x, \quad 0 \leq \sigma<+\infty .
$$

From (7) it follows that for every $\varepsilon>0$ and all $\sigma \geq 0$

$$
\frac{\varepsilon \sigma}{1+\varepsilon} \Lambda\left(\frac{\sigma}{1+\varepsilon}, F\right) \leq \ln \mu(\sigma, F)-\ln \mu(0, F) \leq \sigma \Lambda(\sigma, F) .
$$

Hence $\ln \mu(\sigma, F) \geq \Lambda\left(\frac{\sigma}{1+\varepsilon}, F\right)$ for all $\sigma>0$ large enough and, thus,

$$
\begin{aligned}
& \varrho_{\alpha, \beta}[\Lambda, F]=\varlimsup_{\sigma \rightarrow+\infty} \frac{\alpha(\Lambda(\sigma, F))}{\beta(\sigma)} \leq \varlimsup_{\sigma \rightarrow+\infty} \frac{\alpha(\mu((1+\varepsilon) \sigma, F))}{\beta((1+\varepsilon) \sigma)} \varlimsup_{\sigma \rightarrow+\infty} \frac{\beta((1+\varepsilon) \sigma)}{\beta(\sigma)}, \\
& \lambda_{\alpha, \beta}[\Lambda, F]=\varliminf_{\sigma \rightarrow+\infty} \frac{\alpha(\Lambda(\sigma, F))}{\beta(\sigma)} \leq \varliminf_{\sigma \rightarrow+\infty} \frac{\alpha(\mu((1+\varepsilon) \sigma, F))}{\beta((1+\varepsilon) \sigma)} \varlimsup_{\sigma \rightarrow+\infty} \frac{\beta((1+\varepsilon) \sigma)}{\beta(\sigma)} .
\end{aligned}
$$

Therefore, $\varrho_{\alpha, \beta}[\Lambda, F] \leq \varrho_{\alpha, \beta}[\ln \mu, F] B(\varepsilon)$ and $\lambda_{\alpha, \beta}[\Lambda, F] \leq \lambda_{\alpha, \beta}[\ln \mu, F] B(\varepsilon)$, where in view of condition $\beta \in L^{0}$ we get [11] $B(\varepsilon)=\varlimsup_{\sigma \rightarrow+\infty} \frac{\beta((1+\varepsilon) \sigma)}{\beta(\sigma)} \rightarrow 1$ as $\varepsilon \rightarrow 0$, and thus, $\varrho_{\alpha, \beta}[\Lambda, F] \leq \varrho_{\alpha, \beta}[\ln \mu, F]$ and $\lambda_{\alpha, \beta}[\Lambda, F] \leq \lambda_{\alpha, \beta}[\ln \mu, F]$.

On the other hand, if on the contrary $\varrho_{\alpha, \beta}[\Lambda, F]<\varrho_{\alpha, \beta}[\ln \mu, F]$ then for every $\varrho \in\left(\varrho_{\alpha, \beta}[\Lambda, F], \varrho_{\alpha, \beta}[\ln \mu, F]\right)$ and all $\sigma \geq \sigma_{0}(\varrho)$ we have $\Lambda(\sigma, F) \leq \alpha^{-1}(\varrho \beta(\sigma))$ and, 
thus, $\ln \mu(\sigma, F) \leq(1+o(1)) \sigma \alpha^{-1}(\varrho \beta(\sigma))$ as $\sigma \rightarrow+\infty$, i.e.

$$
\begin{aligned}
\alpha(\ln \mu(\sigma, F)) & \leq(1+o(1)) \alpha\left(\sigma \alpha^{-1}(\varrho \beta(\sigma))\right)= \\
& =(1+o(1)) \alpha\left(\exp \left\{\ln \sigma+\ln \alpha^{-1}(\varrho \beta(\sigma))\right\}\right) \leq \\
& \leq(1+o(1)) \alpha\left(\exp \left\{2 \max \left\{\ln \sigma, \ln \alpha^{-1}(\varrho \beta(\sigma))\right\}\right\}\right)= \\
& =(1+o(1)) \alpha\left(\exp \left\{\max \left\{\ln \sigma, \ln \alpha^{-1}(\varrho \beta(\sigma)\}\right\}\right)=\right. \\
& =(1+o(1)) \max \{\alpha(\sigma), \varrho \beta(\sigma)\}) \leq \\
& \leq(1+o(1))(\alpha(\sigma)+\varrho \beta(\sigma)\})= \\
& =(1+o(1)) \varrho \beta(\sigma)), \quad \sigma \rightarrow+\infty,
\end{aligned}
$$

whence $\varrho_{\alpha, \beta}[\ln \mu, F] \leq \varrho$, which is impossible. Thus, $\varrho_{\alpha, \beta}[\ln \mu, F]=\varrho_{\alpha, \beta}[\Lambda, F]$. The proof of the equality $\lambda_{\alpha, \beta}[\ln \mu, F]=\lambda_{\alpha, \beta}[\Lambda, F]$ is similar.

Lemma 3. If $\alpha \in L^{0}$ and $\beta \in L^{0}$ then $\varrho_{\alpha, \beta}[F]=\varrho_{\alpha, \beta}\left[F^{\prime}\right]$ and $\lambda_{\alpha, \beta}[F]=\lambda_{\alpha, \beta}\left[F^{\prime}\right]$.

Proof. Since [7] for $\sigma<+\infty$ and $0<\delta(\sigma)<+\infty$

$$
M\left(\sigma, F^{\prime}\right) \leq \frac{M(\sigma+\delta(\sigma), F)}{\delta(\sigma)}
$$

and for $\sigma_{0}<\sigma$

$$
M(\sigma, F) \leq\left(\sigma-\sigma_{0}\right) M\left(\sigma, F^{\prime}\right)+M\left(\sigma_{0}, F\right),
$$

using $\delta(\sigma)=1$ and $\sigma_{0}=0$ we have

$$
(1+o(1)) \ln M(\sigma, F) \leq \ln M\left(\sigma, F^{\prime}\right) \leq \ln M(\sigma+1, F), \quad \sigma \rightarrow+\infty,
$$

because for every entire Dirichlet series $\ln \sigma=o(\ln M(\sigma, F))$ as $\sigma \rightarrow+\infty$. Since $\alpha \in L^{0}$ and $\beta \in L^{0}$, we get $\varrho_{\alpha, \beta}[\ln \mu]=\varrho_{\alpha, \beta}[\Lambda]$ and $\lambda_{\alpha, \beta}[\ln \mu]=\lambda_{\alpha, \beta}[\Lambda]$.

Using Lemma 1 we prove the following statement.

Proposition 3. Let the functions $\alpha, \beta$ and the sequence $\left(\lambda_{k}\right)$ satisfy the conditions of Lemma 1, $A[F]=+\infty$ and $-\infty<A[G] \leq \bar{A}[G]<+\infty$. Then $\varrho_{\alpha, \beta}[F * G]=\varrho_{\alpha, \beta}[F]$ and if, moreover, $\alpha\left(\lambda_{k+1}\right) \sim \alpha\left(\lambda_{k}\right), \kappa_{k}[F] \nearrow+\infty$ and $\kappa_{k}[G] \nearrow A[G]$ as $k_{0} \leq k \rightarrow \infty$ then $\lambda_{\alpha, \beta}[F * G]=\lambda_{\alpha, \beta}[F]$

Proof. Clearly, if $A[F]=+\infty$ then $\frac{1}{\lambda_{k}} \ln \frac{1}{\left|f_{k}\right|} \rightarrow+\infty$ as $k \rightarrow \infty$. On the other hand, since $-\infty<A[G] \leq \bar{A}[G]<+\infty$, we have $\frac{1}{\lambda_{k}} \ln \frac{1}{\left|g_{k}\right|}=O(1)$ as $k \rightarrow \infty$. Therefore,

$$
\begin{aligned}
\beta\left(\frac{1}{\lambda_{k}} \ln \frac{1}{\left|f_{k}\right|}+\frac{1}{\lambda_{k}} \ln \frac{1}{\left|g_{k}\right|}\right) & =\beta\left(\frac{1}{\lambda_{k}} \ln \frac{1}{\left|f_{k}\right|}+O(1)\right)= \\
& =\beta\left(\frac{1+o(1)}{\lambda_{k}} \ln \frac{1}{\left|f_{k}\right|}\right)= \\
& =(1+o(1)) \beta\left(\frac{1}{\lambda_{k}} \ln \frac{1}{\left|f_{k}\right|}\right), \quad k \rightarrow \infty,
\end{aligned}
$$


and by Lemma 1

$$
\varrho_{\alpha, \beta}[F * G]=\varlimsup_{k \rightarrow \infty} \frac{\alpha\left(\lambda_{k}\right)}{\beta\left(\frac{1}{\lambda_{k}} \ln \frac{1}{\left|f_{k} g_{k}\right|}\right)}=\varlimsup_{k \rightarrow \infty} \frac{\alpha\left(\lambda_{k}\right)}{\beta\left(\frac{1}{\lambda_{k}} \ln \frac{1}{\left|f_{k}\right|}\right)}=\varrho_{\alpha, \beta}[F * G]
$$

and similarly $\lambda_{\alpha, \beta}[F * G]=\lambda_{\alpha, \beta}[F]$.

Lemma 3 implies the following statement.

Proposition 4. If $\alpha \in L^{0}$ and $\beta \in L^{0}$ then

$$
\varrho_{\alpha, \beta}[F * G]=\varrho_{\alpha, \beta}\left[(F * G)^{(n)}\right]=\varrho_{\alpha, \beta}\left[F^{(n)} * G^{(n)}\right]
$$

and

for each $n \geq 1$.

$$
\lambda_{\alpha, \beta}[F * G]=\lambda_{\alpha, \beta}\left[(F * G)^{(n)}\right]=\lambda_{\alpha, \beta}\left[F^{(n)} * G^{(n)}\right]
$$

Indeed, by Lemma 3 we have that

$$
\varrho_{\alpha, \beta}[F * G]=\varrho_{\alpha, \beta}\left[(F * G)^{\prime}\right] \quad \text { and } \quad \lambda_{\alpha, \beta}[F * G]=\lambda_{\alpha, \beta}\left[(F * G)^{\prime}\right],
$$

that is

$$
\varrho_{\alpha, \beta}[F * G]=\varrho_{\alpha, \beta}\left[(F * G)^{(n)}\right] \quad \text { and } \quad \lambda_{\alpha, \beta}[F * G]=\lambda_{\alpha, \beta}\left[(F * G)^{(n)}\right]
$$

for each $n \geq 1$, and since $F^{(n)} * G^{(n)}=(F * G)^{(2 n)}$, we have that

$$
\varrho_{\alpha, \beta}[F * G]=\varrho_{\alpha, \beta}\left[F^{(n)} * G^{(n)}\right] \quad \text { and } \quad \lambda_{\alpha, \beta}[F * G]=\lambda_{\alpha, \beta}\left[F^{(n)} * G^{(n)}\right] .
$$

\section{Behavior of the maximal terms of Hadamard compositions}

The following is the main result in the section.

Theorem 1. Let $\alpha\left(e^{x}\right) \in L_{s i}, \beta \in L^{0}, \frac{d \beta^{-1}(c \alpha(x))}{d \ln x}=O(1)$ as $x \rightarrow+\infty$ and (4) holds for each $c \in(0,+\infty)$. If $A[F]=+\infty$ and $-\infty<A[G] \leq \bar{A}[G]<+\infty$ then for $n \in \mathbb{Z}_{+}$, $m \in \mathbb{N}$ and $m>n$

$$
\varlimsup_{\sigma \rightarrow+\infty} \frac{1}{\beta(\sigma)} \alpha\left(\frac{\mu\left(\sigma,(F * G)^{(m)}\right)}{\mu\left(\sigma,(F * G)^{(n)}\right)}\right)=\varrho_{\alpha \beta}[F] .
$$

If, moreover, $\alpha\left(\lambda_{k+1}\right) \sim \alpha\left(\lambda_{k}\right), \kappa_{k}[F] \nearrow+\infty$ and $\kappa_{k}[G] \nearrow A[G]$ as $k_{0} \leq k \rightarrow \infty$ then

$$
\varliminf_{\sigma \rightarrow+\infty} \frac{1}{\beta(\sigma)} \alpha\left(\frac{\mu\left(\sigma,(F * G)^{(m)}\right)}{\mu\left(\sigma,(F * G)^{(n)}\right)}\right)=\lambda_{\alpha \beta}[F] .
$$

Proof. The following inequalities proved in [7] play an important role in the proof of Theorem 1

$$
\Lambda^{m-n}\left(\sigma,(F * G)^{(n)}\right) \leq \frac{\mu\left(\sigma,(F * G)^{(m)}\right)}{\mu\left(\sigma,(F * G)^{(n)}\right)} \leq \Lambda^{m-n}\left(\sigma,(F * G)^{(m)}\right)
$$

for $\sigma<\sigma_{a}[F * G]$. Since $\alpha\left(e^{x}\right) \in L_{s i}$, we have

$$
\begin{aligned}
\alpha\left(\Lambda^{m-n}\left(\sigma,(F * G)^{(n)}\right)\right) & =\alpha\left(\exp \left\{(m-n) \ln \Lambda\left(\sigma,(F * G)^{(n)}\right)\right\}\right)= \\
& =(1+o(1)) \alpha\left(\exp \left\{\ln \Lambda\left(\sigma,(F * G)^{(n)}\right)\right\}\right)= \\
& =(1+o(1)) \alpha\left(\Lambda\left(\sigma,(F * G)^{(n)}\right)\right), \quad \sigma \rightarrow+\infty
\end{aligned}
$$


and, therefore, (13) implies

$$
\alpha\left(\Lambda\left(\sigma,(F * G)^{(n)}\right)\right) \leq(1+o(1)) \alpha\left(\frac{\mu\left(\sigma,(F * G)^{(m)}\right)}{\mu\left(\sigma,(F * G)^{(n)}\right)}\right) \leq \alpha\left(\Lambda\left(\sigma,(F * G)^{(m)}\right)\right)
$$

as $\sigma \rightarrow+\infty$, whence

$$
\begin{aligned}
\varrho_{\alpha \beta}\left[\Lambda,(F * G)^{(n)}\right] & \leq \varlimsup_{\sigma \rightarrow+\infty} \frac{1}{\beta(\sigma)} \alpha\left(\frac{\mu\left(\sigma,(F * G)^{(m)}\right)}{\mu\left(\sigma,(F * G)^{(n)}\right)}\right) \leq \\
& \leq \varrho_{\alpha \beta}\left[\Lambda,(F * G)^{(m)}\right]
\end{aligned}
$$

and

$$
\begin{aligned}
\lambda_{\alpha \beta}\left[\Lambda,(F * G)^{(n)}\right] & \leq \lim _{\sigma \rightarrow+\infty} \frac{1}{\beta(\sigma)} \alpha\left(\frac{\mu\left(\sigma,(F * G)^{(m)}\right)}{\mu\left(\sigma,(F * G)^{(n)}\right)}\right) \leq \\
& \leq \lambda_{\alpha \beta}\left[\Lambda,(F * G)^{(m)}\right] .
\end{aligned}
$$

We remark that the condition $\frac{d \beta^{-1}(c \alpha(x))}{d \ln x}=O(1)$ as $x \rightarrow+\infty$ for each $c \in(0,+\infty)$ implies the condition $\alpha(x)=o(\beta(x))$ as $x \rightarrow+\infty$. Therefore, applying Lemma 2, Lemma 1, Proposition 4 and Proposition 3 consequently, we obtain $\varrho_{\alpha \beta}\left[\Lambda,(F * G)^{(n)}\right]=$ $=\varrho_{\alpha \beta}\left[\ln \mu,(F * G)^{(n)}\right]=\varrho_{\alpha \beta}\left[(F * G)^{(n)}\right]=\varrho_{\alpha \beta}[F * G]=\varrho_{\alpha \beta}[F]$ and similarly $\lambda_{\alpha \beta}\left[\Lambda,(F * G)^{(n)}\right]=\lambda_{\alpha \beta}[F]$. Therefore, from (14) and (15) we get (11) and (12).

Choosing $m=2 n$ we obtain the following corollary.

Corollary 1. Let the functions $\alpha, \beta$ and the sequence $\left(\lambda_{k}\right)$ satisfy the conditions of Theorem 1, $A[F]=+\infty$ and $-\infty<A[G] \leq \bar{A}[G]<+\infty$ then for $n \in \mathbb{N}$

$$
\varlimsup_{\sigma \rightarrow+\infty} \frac{1}{\beta(\sigma)} \alpha\left(\frac{\mu\left(\sigma, F^{(n)} * G^{(n)}\right)}{\mu\left(\sigma,(F * G)^{(n)}\right)}\right)=\varrho_{\alpha \beta}[F] .
$$

If, moreover, $\alpha\left(\lambda_{k+1}\right) \sim \alpha\left(\lambda_{k}\right), \kappa_{k}[F] \nearrow+\infty$ and $\kappa_{k}[G] \nearrow A[G]$ as $k_{0} \leq k \rightarrow \infty$ then

$$
\varliminf_{\sigma \rightarrow+\infty} \frac{1}{\beta(\sigma)} \alpha\left(\frac{\mu\left(\sigma, F^{(n)} * G^{(n)}\right)}{\mu\left(\sigma,(F * G)^{(n)}\right)}\right)=\lambda_{\alpha \beta}[F] .
$$

\section{HAdAMARd COMpositions of the Finite $R$-ORder}

If we choose $\alpha(x)=\ln x$ and $\beta(x)=x$ for $x \geq 3$ then from (3) we obtain the definition of the $R$-order

$$
\varrho_{R}[F]:=\varlimsup_{\sigma \rightarrow+\infty} \frac{\ln \ln M(\sigma, F)}{\sigma}
$$

and the lower $R$-order

$$
\lambda_{R}[F]:=\varliminf_{\sigma \rightarrow+\infty} \frac{\ln \ln M(\sigma, F)}{\sigma}
$$

introduced by J. Ritt [12] for a function $F \in S(\Lambda,+\infty)$.

The functions $\alpha(x)=\ln x$ and $\beta(x)=x$ satisfy the conditions of Lemmas 1 and 3 and do not satisfy the condition $\alpha\left(e^{x}\right) \in L_{s i}$ of Lemma 2. But it follows from (8) that $\varrho_{R}[\Lambda, F]=\varrho_{R}[\ln \mu, F]$ and $\lambda_{R}[\Lambda, F]=\lambda_{R}[\ln \mu, F]$. Therefore, as in the proof of 
Theorem 1, we have $\varrho_{R}\left[\Lambda,(F * G)^{(n)}\right]=\varrho_{R}[F]$ and $\lambda_{R}\left[\Lambda,(F * G)^{(n)}\right]=\lambda_{R}[F]$. On the other hand, from (13) we get

$$
\begin{aligned}
(m-n) \ln \Lambda\left(\sigma,(F * G)^{(n)}\right) & \leq \ln \frac{\mu\left(\sigma,(F * G)^{(m)}\right)}{\mu\left(\sigma,(F * G)^{(n)}\right)} \leq \\
& \leq(m-n) \ln \Lambda^{m-n}\left(\sigma,(F * G)^{(m)}\right)
\end{aligned}
$$

and, thus, the following theorem is true.

Theorem 2. If $A[F]=+\infty,-\infty<A[G] \leq \bar{A}[G]<+\infty)$, and $\ln k=o\left(\lambda_{k} \ln \lambda_{k}\right)$ as $k \rightarrow \infty$. Then for $n \in \mathbb{Z}_{+}, m \in \mathbb{N}$ and $m>n$

$$
\varlimsup_{\sigma \rightarrow+\infty} \frac{1}{\sigma} \ln \frac{\mu\left(\sigma,(F * G)^{(m)}\right)}{\mu\left(\sigma,(F * G)^{(n)}\right)}=(m-n) \varrho_{R}[F] .
$$

If, moreover, $\ln \lambda_{k+1} \sim \ln \lambda_{k}, \kappa_{k}[F] \nearrow+\infty$ and $\kappa_{k}[G] \nearrow A[G]$ as $k_{0} \leq k \rightarrow \infty$ then

$$
\varliminf_{\sigma \rightarrow+\infty} \frac{1}{\sigma} \ln \frac{\mu\left(\sigma,(F * G)^{(m)}\right)}{\mu\left(\sigma,(F * G)^{(n)}\right)}=(m-n) \lambda_{R}[F] .
$$

If we choose $m=2 n+2$ then from Theorem 2 we obtain the following analogue of the above-mentioned result of M.K. Sen.

Corollary 2. $A[F]=+\infty,-\infty<A[G] \leq \bar{A}[G]<+\infty$, and $\ln k=o\left(\lambda_{k} \ln \lambda_{k}\right)$ as $k \rightarrow \infty$. Then for $n \in \mathbb{Z}_{+}$

$$
\varlimsup_{\sigma \rightarrow+\infty} \frac{1}{\sigma} \ln \frac{\mu\left(\sigma, F^{(n+1)} * G^{(n+1)}\right)}{\mu\left(\sigma,(F * G)^{(n)}\right)}=(n+2) \varrho_{R}[F] .
$$

If, moreover, $\ln \lambda_{k+1} \sim \ln \lambda_{k}, \kappa_{k}[F] \nearrow+\infty$, and $\kappa_{k}[G] \nearrow A[G]$ as $k_{0} \leq k \rightarrow \infty$ then

$$
\varliminf_{\sigma \rightarrow+\infty} \frac{1}{\sigma} \ln \frac{\mu\left(\sigma, F^{(n+1)} * G^{(n+1)}\right)}{\mu\left(\sigma,(F * G)^{(n)}\right)}=(n+2) \lambda_{R}[F] .
$$

Let now $0<\varrho_{R}[F]<+\infty$. If we choose $\alpha(x)=x$ and $\beta(x)=\exp \left\{\varrho_{R}[F] x\right\}$ for $x \geq 0$ then from (3) we obtain the definition of the $R$-type,

$$
T_{R}[F]=\varlimsup_{\sigma \rightarrow+\infty} \frac{\ln M(\sigma, F)}{\exp \left\{\varrho_{R}[F] \sigma\right\}},
$$

and the lover $R$-type,

$$
t_{R}[F]=\varliminf_{\sigma \rightarrow+\infty} \frac{\ln M(\sigma, F)}{\exp \left\{\varrho_{R}[F] \sigma\right\}} .
$$

It is clear that the functions $\alpha(x)=x$ and $\beta(x)=\exp \left\{\varrho_{R}[F] x\right\}$ do not satisfy the conditions of Lemma 1, but the following lemma is true (see for example [10], [12], [13]).

Lemma 4. If $F \in(\Lambda,+\infty)$ and $\ln k=o\left(\lambda_{k}\right)$ as $k \rightarrow \infty$ then

$$
T_{R}[F]=T_{R}[\ln \mu, F]=\varlimsup_{k \rightarrow \infty} \frac{\lambda_{k}}{e \varrho_{R}[F]}\left|f_{k}\right|^{\varrho_{R}[F] / \lambda_{k}} .
$$

If, moreover, $\lambda_{k+1} \sim \lambda_{k}$ and $\kappa_{k}[F] \nearrow+\infty$ as $k_{0} \leq k \rightarrow \infty$ then

$$
t_{R}[F]=t_{R}[\ln \mu, F]=\varliminf_{k \rightarrow \infty} \frac{\lambda_{k}}{e \varrho_{R}[F]}\left|f_{k}\right|^{\varrho_{R}[F] / \lambda_{k}} .
$$


The following lemma indicates the connection between the growth of $\ln \mu(\sigma, F)$ and $\Lambda(\sigma, F)$ in terms of $R$-types.

Lemma 5. Let $F \in(\Lambda,+\infty)$ and $\ln k=o\left(\lambda_{k}\right)$ as $k \rightarrow \infty$. Then

$$
\frac{T_{R}[\Lambda, F]}{e \varrho_{R}[F]} \leq T_{R}[\ln \mu, F] \leq \frac{T_{R}[\Lambda, F]}{\varrho_{R}[F]}
$$

and

$$
\frac{t_{R}[\Lambda, F]}{e \varrho_{R}[F]} \leq t_{R}[\ln \mu, F] \leq \frac{T_{R}[\Lambda, F]}{\varrho_{R}[F]} \ln \frac{e \varrho_{R}[F] T_{R}[\ln \mu, F]}{T_{R}[\Lambda, F]} .
$$

Proof. From (7) for $\sigma \geq 1 / \varrho_{R}[F]$ we have

$$
\ln \mu(\sigma, F)-\ln \mu(0, F) \geq \int_{\sigma-1 / \varrho_{R}[F]}^{\sigma} \Lambda(x) d x \geq \frac{\Lambda\left(\sigma-1 / \varrho_{R}[F]\right)}{\varrho_{R}[F]}
$$

i.e.,

$$
T_{R}[\ln \mu, F] \geq \varlimsup_{\sigma \rightarrow+\infty} \frac{\Lambda\left(\sigma-1 / \varrho_{R}[F]\right)}{\varrho_{R}[F] \exp \left\{\varrho_{R}[F] \sigma\right\}}=\varlimsup_{\sigma \rightarrow+\infty} \frac{\Lambda\left(\sigma-1 / \varrho_{R}[F]\right)}{e \varrho_{R}[F] \exp \left\{\varrho_{R}[F]\left(\sigma-1 / \varrho_{R}[F]\right)\right\}},
$$

whence $T_{R}[\ln \mu, F] \geq \frac{T_{R}[\Lambda, F]}{e \varrho_{R}[F]}$. Similarly, $t_{R}[\ln \mu, F] \geq \frac{t_{R}[\Lambda, F]}{e \varrho_{R}[F]}$. Thus, the inequalities on the left side in (17) and (18) are proved.

On the other hand, if $T_{R}[\Lambda, F]<+\infty$ then $\Lambda(\sigma) \leq T \exp \left\{\varrho_{R}[F] \sigma\right\}$ for every $T>T_{R}[\Lambda . F]$ and all $\sigma \geq \sigma_{0}(T)$. Therefore,

$$
\begin{aligned}
\ln \mu(\sigma, F)-\ln \mu\left(\sigma_{0}(T), F\right) & \leq T \int_{\sigma_{0}(T)}^{\sigma} \exp \left\{\varrho_{R}[F] x\right\} d x= \\
& =\frac{T}{\varrho_{R}[F]}\left(\exp \left\{\varrho_{R}[F] \sigma\right\}-\exp \left\{\varrho_{R}[F] \sigma_{0}(T)\right\}\right),
\end{aligned}
$$

whence $T_{R}[\ln \mu, F] \leq T / \varrho_{R}[F]$, i.e. in view of the arbitrariness of $T$ we get $T_{R}[\ln \mu, F] \leq$ $T_{R}[\Lambda, F] / \varrho_{R}[F]$.

Finally, suppose that $t_{R}[\ln \mu, F]>0$ and $T_{R}[\Lambda, F]>0$. Then for every $t \in\left(0, t_{R}[\ln \mu, F]\right)$ and $T \in\left(0, T_{R}[\Lambda, F]\right)$ there exists an unbounded set $E \subset[0,+\infty)$ such that $\ln \mu(\sigma, F) \geq t \exp \{\varrho[F] \sigma\}$ and $\Lambda(\sigma) \geq T \exp \left\{\varrho_{R}[F] \sigma\right\}$. Therefore, for $\sigma^{*} \in E$ and $\sigma>\sigma^{*}$

$$
\begin{aligned}
\ln \mu(\sigma, F) & =\ln \mu\left(\sigma^{*}, F\right)+\int_{\sigma^{*}}^{\sigma} \Lambda(x, F) d x \\
& \geq \ln \mu\left(\sigma^{*}, F\right)+\Lambda\left(\sigma^{*}, F\right) \int_{\sigma^{*}}^{\sigma} d x \geq \\
& \geq t \exp \left\{\varrho_{R}[F] \sigma^{*}\right\}+\left(\sigma-\sigma^{*}\right) T \exp \left\{\varrho_{R}[F] \sigma^{*}\right\} .
\end{aligned}
$$


Therefore,

$$
\frac{\ln \mu(\sigma, F)}{\exp \left\{\varrho_{R}[F] \sigma\right\}} \geq \frac{t+\left(\sigma-\sigma^{*}\right) T}{\exp \left\{\varrho_{R}[F]\left(\sigma-\sigma^{*}\right)\right\}} .
$$

Since the maximum of the function $\varphi(x)=\frac{t+T x}{\exp \left\{\varrho_{R}[F] x\right\}}$ is reached at the point $x=\frac{T-t \varrho_{R}[F]}{T \varrho_{R}[F]}$, we obtain $T_{R}[\ln \mu] \geq \frac{T}{e \varrho_{R}[F]} \exp \left\{\frac{\varrho_{R}[F] t}{T}\right\}$ and in view of the arbitrariness of $t$ and $T$ we get

$$
T_{R}[\ln \mu, F] \geq \frac{T_{R}[\Lambda, F]}{e \varrho_{R}[F]} \exp \left\{\frac{\varrho_{R}[F] t_{R}[\ln \mu, F]}{T_{R}[\Lambda, F]}\right\},
$$

whence the right side of (18) follows. The proof of Lemma 5 is complete.

Lemma 6. For every entire Dirichlet series (1) $T_{R}[F]=T_{R}\left[F^{\prime}\right]$ and $t_{R}[F]=t_{R}\left[F^{\prime}\right]$.

Proof. Choosing $\delta(\sigma)=1 /(\sigma+1)$ for $\sigma \geq 0$ from (9) we obtain

$$
\begin{aligned}
\frac{\ln M\left(\sigma, F^{\prime}\right)}{\exp \left\{\sigma \varrho_{R}[F]\right\}} & \leq \frac{\ln M\left(\sigma+1 /(\sigma+1), F^{\prime}\right)+\ln (\sigma+1)}{\exp \left\{\sigma \varrho_{R}[F]\right\}}= \\
& =\frac{\ln M\left(\sigma+1 /(\sigma+1), F^{\prime}\right)}{\exp \left\{(\sigma+1 /(\sigma+1)) \varrho_{R}[F]\right\}} \exp \left\{\frac{\varrho_{R}[F]}{\sigma+1}\right\}+\frac{\ln (\sigma+1)}{\exp \left\{\sigma \varrho_{R}[F]\right\}},
\end{aligned}
$$

whence $T_{R}\left[F^{\prime}\right] \leq T_{R}[F]$ and $t_{R}\left[F^{\prime}\right] \leq t_{R}[F]$. On the other hand, in view of (10) $\ln M(\sigma, F) \leq(1+o(1)) \ln M(\sigma, F)$ as $\sigma \rightarrow+\infty$, whence $T_{R}[F] \leq T_{R}\left[F^{\prime}\right]$ and $t_{R}[F] \leq t_{R}\left[F^{\prime}\right]$.

Using Lemma 4 we prove the following statement.

Proposition 5. Let $A[F]=+\infty,-\infty<A[G] \leq \bar{A}[G]<+\infty$ and $\ln k=o\left(\lambda_{k}\right)$ as $k \rightarrow \infty$. Then

$$
T_{R}[F] \exp \left\{-\bar{A}[G] \varrho_{R}[F]\right\} \leq T_{R}[F * G] \leq T_{R}[F] \exp \left\{-A[G] \varrho_{R}[F]\right\}
$$

and if, moreover, $\lambda_{k+1} \sim \lambda_{k}, \kappa_{k}[F] \nearrow+\infty$ and $\kappa_{k}[G] \nearrow A[G]$ as $k_{0} \leq k \rightarrow \infty$ then

$$
t_{R}[F] \exp \left\{-\bar{A}[G] \varrho_{R}[F]\right\} \leq t_{R}[F * G] \leq t_{R}[F] \exp \left\{-A[G] \varrho_{R}[F]\right\} .
$$

Proof. By Proposition $3 \varrho_{R}[F * G]=\varrho_{R}[F]$. Therefore, by Lemma 4

$$
\begin{aligned}
T_{R}[F * G] & =\left.\varlimsup_{k \rightarrow \infty} \frac{\lambda_{k}}{e \varrho_{R}[F * G}|| f_{k} g_{k}\right|^{\varrho_{R}[F * G] / \lambda_{k}}= \\
& =\varlimsup_{k \rightarrow \infty} \frac{\lambda_{k}}{e \varrho_{R}[F]}\left|f_{k}\right|^{\varrho_{R}[F] / \lambda_{k}} \exp \left\{-\varrho_{R}[F] \frac{1}{\lambda_{k}} \ln \frac{1}{\left|g_{k}\right|}\right\},
\end{aligned}
$$

whence

$$
\begin{aligned}
T_{R}[F * G] & \leq \varlimsup_{k \rightarrow \infty} \frac{\lambda_{k}}{e \varrho_{R}[F]}\left|f_{k}\right|^{\varrho_{R}[F] / \lambda_{k}} \varlimsup_{k \rightarrow \infty} \exp \left\{-\varrho_{R}[F] \frac{1}{\lambda_{k}} \ln \frac{1}{\left|g_{k}\right|}\right\}= \\
& =T_{R}[F] \exp \left\{-\varrho_{R}[F] \underset{k \rightarrow \infty}{\lim _{i}} \frac{1}{\lambda_{k}} \ln \frac{1}{\left|g_{k}\right|}\right\}= \\
& =T_{R}[F] \exp \left\{-A[G] \varrho_{R}[F]\right\} .
\end{aligned}
$$


and

$$
\begin{aligned}
& T_{R}[F * G] \geq \varlimsup_{k \rightarrow \infty} \frac{\lambda_{k}}{e \varrho_{R}[F]}\left|f_{k}\right|_{R}[F] / \lambda_{k} \\
& \lim _{k \rightarrow \infty} \exp \left\{-\varrho_{R}[F] \frac{1}{\lambda_{k}} \ln \frac{1}{\left|g_{k}\right|}\right\}= \\
&=T_{R}[F] \exp \left\{-\varrho_{R}[F] \varlimsup_{k \rightarrow \infty} \frac{1}{\lambda_{k}} \ln \frac{1}{\left|g_{k}\right|}\right\}= \\
&=T_{R}[F] \exp \left\{-\bar{A}[G] \varrho_{R}[F]\right\},
\end{aligned}
$$

i.e. we get (19). The proof of (20) is similar.

Finally, Lemma 6 implies the following statement.

Proposition 6. The equalities

$$
T_{R}[F * G]=T_{R}\left[(F * G)^{(n)}\right]=T_{R}\left[F^{(n)} * G^{(n)}\right]
$$

and

$$
t_{R}[F * G]=t_{R}\left[(F * G)^{(n)}\right]=t_{R}\left[F^{(n)} * G^{(n)}\right]
$$

are true for each $n \geq 1$.

Therefore, the following theorem is true.

Theorem 3. Let $A[F]=+\infty,-\infty<A[G] \leq \bar{A}[G]<+\infty$ and $\ln k=o\left(\lambda_{k}\right)$ as $k \rightarrow \infty$. Then for $n \in \mathbb{Z}_{+}, m \in \mathbb{N}$ and $m>n$

$$
\begin{aligned}
\frac{\varrho_{R}[F] T_{R}[F * G]}{\exp \left\{\bar{A}[G] \varrho_{R}[F]\right\}} & \leq \varlimsup_{\sigma \rightarrow+\infty} \frac{1}{\exp \left\{\varrho_{R}[F] \sigma\right\}} \sqrt[m-n]{\frac{\mu\left(\sigma,(F * G)^{(m)}\right)}{\mu\left(\sigma,(F * G)^{(n)}\right)}} \leq \\
& \leq \frac{e \varrho_{R}[F] T_{R}[F * G]}{\exp \left\{A[G] \varrho_{R}[F]\right\}}
\end{aligned}
$$

Proof. From (13) it follows that

$$
\begin{aligned}
T_{R}\left[\Lambda,(F * G)^{(n)}\right] & \left.\leq \varlimsup_{\sigma \rightarrow+\infty} \frac{1}{\exp \left\{\varrho_{R}[F] \sigma\right\}} \sqrt[m-n]{\frac{\mu\left(\sigma,(F * G)^{(m)}\right)}{\mu\left(\sigma,(F * G)^{(n)}\right)}}\right) \leq \\
& \leq T_{R}\left[\Lambda,(F * G)^{(m)}\right] .
\end{aligned}
$$

Using Proposition 6, Lemmas 5 and 4 from (22) we get (21).

Remark 1. Similarly, we can prove that if the conditions of Theorem 3 are satisfied and, moreover, $\lambda_{k+1} \sim \lambda_{k}, \kappa_{k}[F] \nearrow+\infty$ and $\kappa_{k}[G] \nearrow A[G]$ as $k_{0} \leq k \rightarrow \infty$ then

$$
\varliminf_{\sigma \rightarrow+\infty} \frac{1}{\exp \left\{\varrho_{R}[F] \sigma\right\}} \sqrt[m-n]{\frac{\mu\left(\sigma,(F * G)^{(m)}\right)}{\mu\left(\sigma,(F * G)^{(n)}\right)}} \leq \frac{e \varrho_{R}[F] t_{R}[F * G]}{\exp \left\{A[G] \varrho_{R}[F]\right\}}
$$

We were not able to obtain a lower estimate for this $\underline{\lim }$, because there is no such an estimate for $t_{R}(\Lambda)$. 


\section{Hadamard Compositions of the Finite LOGARITHMic ORder}

In the theory of entire Dirichlet series, the logarithmic order

$$
\varrho_{l}[F]:==\varlimsup_{\sigma \rightarrow+\infty} \frac{\ln \ln M(\sigma, F)}{\ln \sigma}
$$

and lower order

$$
\lambda_{l}[F]:=\varliminf_{\sigma \rightarrow+\infty} \frac{\ln \ln M(\sigma, F)}{\ln \sigma}
$$

are also used. We remark that $\lambda_{l}[F] \geq 1$ for each entire Dirichlet series.

The function $\alpha(x)=\beta(x)=\ln x$ not hold the condition of Lemma 1 , but the following statement is true [13].

Lemma 7. If $F \in(\Lambda,+\infty)$ and

$$
\varlimsup_{k \rightarrow \infty} \frac{\ln \ln k}{\ln \lambda_{k}}<1
$$

then $\varrho_{l}[F]=\varlimsup_{k \rightarrow \infty} \frac{\ln \lambda_{k}}{\ln \left(\frac{1}{\lambda_{k}} \ln \frac{1}{\left|f_{k}\right|}\right)}+1$. If, moreover, $\ln \lambda_{k+1} \sim \ln \lambda_{k}$ and $\kappa_{k}[F] \nearrow+\infty$ as $k_{0} \leq k \rightarrow \infty$ then $\lambda_{l}[F]=\varliminf_{k \rightarrow \infty} \frac{\ln \lambda_{k}}{\ln \left(\frac{1}{\lambda_{k}} \ln \frac{1}{\left|f_{n}\right|}\right)}+1$.

As in the proof of Proposition 3 using Lemma 7 we get the following statement.

Proposition 7. Let $A[F]=+\infty,-\infty<A[G] \leq \bar{A}[G]<+\infty$ and (23) holds. Then $\varrho_{l}[F * G]=\varrho_{l}[F]$. If, moreover, $\ln \lambda_{k+1} \sim \ln \lambda_{k}, \kappa_{k}[F] \nearrow+\infty$ and $\kappa_{k}[G] \nearrow A[G]$ as $k_{0} \leq k \rightarrow \infty$ then $\lambda_{l}[F * G]=\lambda_{l}[F]$.

From $(9)$ with $\delta(\sigma)=1$ and (10) we obtain $\varrho_{l}\left[F^{\prime}\right]=\varrho_{l}[F]$ and $\lambda_{l}\left[F^{\prime}\right]=\lambda_{l}[F]$. From (8) with $\varepsilon=1$ we obtain

$$
\frac{\sigma}{2} \Lambda\left(\frac{\sigma}{2}, F\right) \leq \ln \mu(\sigma, F)-\ln \mu(0, F) \leq \sigma \Lambda(\sigma)
$$

whence $\varrho_{l}[\ln \mu, F]-1=\varrho_{l}[\Lambda, F]$ and $\lambda_{l}[\ln \mu, F]-1=\lambda_{l}[\Lambda, F]$. Finally, (16) implies the inequalities

$$
\begin{aligned}
(m-n) \varrho_{l}\left(\Lambda,(F * G)^{(n)}\right) & \leq \varlimsup_{\sigma \rightarrow+\infty} \frac{1}{\ln \sigma} \ln \frac{\mu\left(\sigma,(F * G)^{(m)}\right)}{\mu\left(\sigma,(F * G)^{(n)}\right)} \leq \\
& \leq(m-n) \varrho_{l}\left(\Lambda,(F * G)^{(m)}\right)
\end{aligned}
$$

and

$$
\begin{aligned}
(m-n) \lambda_{l}\left(\Lambda,(F * G)^{(n)}\right) & \leq \varlimsup_{\sigma \rightarrow+\infty} \frac{1}{\ln \sigma} \ln \frac{\mu\left(\sigma,(F * G)^{(m)}\right)}{\mu\left(\sigma,(F * G)^{(n)}\right)} \leq \\
& \leq(m-n) \lambda_{l}\left(\Lambda,(F * G)^{(m)}\right) .
\end{aligned}
$$

Therefore, as usual, we arrive at the following theorem. 
Theorem 4. Let $A[F]=+\infty,-\infty<A[G] \leq \bar{A}[G]<+\infty$ and (23) holds. Then for $n \in \mathbb{Z}_{+}, m \in \mathbb{N}$ and $m>n$

$$
\varlimsup_{\sigma \rightarrow+\infty} \frac{1}{\ln \sigma} \ln \frac{\mu\left(\sigma,(F * G)^{(m)}\right)}{\mu\left(\sigma,(F * G)^{(n)}\right)}=(m-n)\left(\varrho_{l}[F]-1\right) .
$$

If, moreover, $\ln \lambda_{k+1} \sim \ln \lambda_{k}, \kappa_{k}[F] \nearrow+\infty$ and $\kappa_{k}[G] \nearrow A[G]$ as $k_{0} \leq k \rightarrow \infty$ then

$$
\varliminf_{\sigma \rightarrow+\infty} \frac{1}{\ln \sigma} \ln \frac{\mu\left(\sigma,(F * G)^{(m)}\right)}{\mu\left(\sigma,(F * G)^{(n)}\right)}=(m-n)\left(\lambda_{l}[F]-1\right) .
$$

\section{REFERENCES}

1. J. Hadamard, Théorème sur les séries entières, Acta Math. 22 (1899), 55-63. DOI: $10.1007 / \mathrm{BF} 02417870$

2. J. Hadamard, La série de Taylor et son prolongement analytique, Évreux, Impr. de C. Hérissey, 1901.

3. L. Bieberbach, Analytische Fortsetzung, Springer, Berlin, 1955.

4. Ю. Ф. Коробейник, Н. Н. Мавроди, Об особъх точках композиции Адамара, Укр. мат. журн. 42 (1990), no. 12, 1711-1713; English version: Yu. F. Korobeinik and N. N. Mavrodi, Singular points of the Hadamard composition, Ukr. Math. J. 42 (1990), no. $12,1545-1547$. DOI: $10.1007 /$ BF 01060828

5. M. K. Sen, On some properties of an integral function $f * g$, Riv. Mat. Univ. Parma, II. Ser. 8 (1967), 317-328.

6. M. K. Sen, On the maximum term of a class of integral functions and its derivatives, Ann. Pol. Math. 22 (1970), 291-298. DOI: 10.4064/ap-22-3-291-298

7. O. M. Mulyava and M. M. Sheremeta, Properties of Hadamard's compositions of derivatives of Dirichlet series, Visn. L'viv. Univ., Ser. Mekh.-Mat. 77 (2012), 157-166.

8. A. F. Leontev, Series of exponents, Nauka, Noscow, 1976 (in Riussian).

9. M. M. Sheremeta, Entire Dirichlet series, ISDO, Kyiv, 1993 (in Ukrainian).

10. M. M. Sheremeta, Asymptotic behaviours of entire functions given by power series and Dirichlet series, Doct. Diss., Kiev, 1987 (in Russian).

11. M. M. Sheremeta, On two classes of positive functions and the belonging to them of main characteristics of entire functions, Mat. Stud. 19 (2003), no. 1, 73-82.

12. J. F. Ritt, On certain points in the theory of Dirichlet series, Am. J. Math. 50 (1928), no. 1, 73-86. DOI: $10.2307 / 2370849$

13. A. R. Reddy, On entire Dirichlet series of zero order, Tohoku Math. J., II Ser. 18 (1966), no. $2,144-155$. DOI: $10.2748 / \mathrm{tmj} / 1178243445$

Стаття: надійшла до редколегї 03.03.2019 доопрацвована 26.04.2019 прийнята до друку 03.02.2020 


\title{
ПРО АДАМАРОВІ КОМПОЗИЦІЇ ЦІЛОГО РЯДУ ДІРІХЛЕ ТА РЯДУ ДІРІХЛЕ, АБСОЛЮТНО ЗБІЖНОГО У ПІВПЛОЩИНІ
}

\author{
Оксана МУЛЯВА ${ }^{1}$, Мирослав ШЕРЕМЕТА $^{2}$ \\ ${ }^{1}$ Киёвсъкий націоналъний університет харчових технологій \\ вул. Володимирівсъка, 68, 01033, Київ \\ e-mail: oksana.m@bigmir.net \\ 2 Лъвівсъкий начіоналъний університет імені Івана Франка, \\ вул. Університетсъка, 1, 79000, Лъвів \\ e-mail: m.m.sheremeta@gmail.com
}

Для степеневих рядів $f(z)=\sum_{k=0}^{\infty} f_{k} z^{k}$ i $g(z)=\sum_{k=0}^{\infty} g_{k} z^{k}$ із радіусами збіжності $R[f]$ і $R[g]$ ряд $(f * g)(z)=\sum_{k=0}^{\infty} f_{k} g_{k} z^{k}$ називається адамаровою композицією. Для $0 \leq r<R[f]$ нехай $\mu_{f}(r)=\max \left\{\left|f_{k}\right| r^{k}: k \geq 0\right\}$ - максимальний член степеневого розвинення функції $f$. Вивчаючи зв'язок між зростанням максимальних членів похідних адамарової композиції двох цілих функцій $f$ та $g$ і адамаровою композицією їх похідних М. Сен зокрема довів, що якщо функція $(f * g)$ має порядок $\varrho$ і нижній порядок $\lambda$, то для кожного $\varepsilon>0$ і всіх $r \geq r_{0}(\varepsilon)$

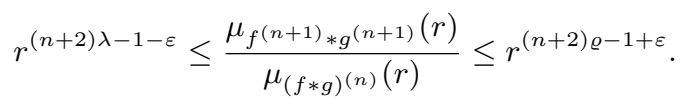

Оскільки ряди Діріхле з додатними зростаючими до $+\infty$ показниками $\epsilon$ прямим узагальненням степеневих рядів, то природно постає питання про подібні результати для адамарової композиції таких рядів. Отже, нехай $\Lambda=\left(\lambda_{k}\right)$ - зростаюча до $+\infty$ послідовність невід'ємних чисел $\left(\lambda_{0}=0\right), \mathrm{i}$ $S(\Lambda, A)$ - клас рядів Діріхле $F(s)=\sum_{k=0}^{\infty} f_{k} \exp \left\{s \lambda_{k}\right\},(s=\sigma+i t)$, з показниками $\Lambda$ і абсцисою абсолютної збіжності $\sigma_{a}[F]=A$. Якщо $F \in\left(\Lambda, A_{1}\right)$ і $G(s)=\sum_{k=0}^{\infty} g_{k} \exp \left\{s \lambda_{k}\right\} \in\left(\Lambda, A_{2}\right)$, то ряд Діріхле

$$
(F * G)(s)=\sum_{k=0}^{\infty} f_{k} g_{k} \exp \left\{s \lambda_{k}\right\}
$$

називається адамаровою композицією функцій $F$ та $G$.

Для ряду Діріхле $F(s)$ з $\sigma_{a}[F]=A[F]=A>-\infty$ для $\sigma<A$ максимальним членом називатимемо $\mu(\sigma, F)=\max \left\{\left|f_{k}\right| \exp \left\{\sigma \lambda_{k}\right\}: k \geq 0\right\}$. Відомо, що для $n \in \mathbb{Z}_{+}, m \in \mathbb{N}$ i $m>n$, якщо $\sigma_{a}[F]=\sigma_{a}[G]=+\infty \mathrm{i} \ln k=o\left(\lambda_{k} \ln \lambda_{k}\right)$ при $k \rightarrow \infty$, то

$$
\varlimsup_{\sigma \rightarrow+\infty} \frac{1}{\sigma} \ln \frac{\mu\left(\sigma,(F * G)^{(m)}\right)}{\mu\left(\sigma,(F * G)^{(n)}\right)}=(m-n) \varrho_{R}[f * G]
$$

i (якщо $\left.\varrho_{R}[f * G]<+\infty\right)$

$$
\varliminf_{\sigma \rightarrow+\infty} \frac{1}{\sigma} \ln \frac{\mu\left(\sigma,(F * G)^{(m)}\right)}{\mu\left(\sigma,(F * G)^{(n)}\right)}=(m-n) \lambda_{R}[f * G],
$$


де $\varrho_{R}[f]$ і $\lambda_{R}[f]$ відповідно $R$-порядок та нижній $R$-порядок цілого ряду Діріхле. Якщо $\sigma_{a}[F]=\sigma_{a}[G]=0 \mathrm{i} \ln k=o\left(\lambda_{k} / \ln \lambda_{k}\right)$ при $k \rightarrow \infty$, то

$$
\varlimsup_{\sigma \uparrow 0}|\sigma| \ln \frac{\mu\left(\sigma,(F * G)^{(m)}\right)}{\mu\left(\sigma,(F * G)^{(n)}\right)}=(m-n) \varrho^{(0)}[f * G]
$$

i

$$
\varliminf_{\sigma \uparrow 0}|\sigma| \ln \frac{\mu\left(\sigma,(F * G)^{(m)}\right)}{\mu\left(\sigma,(F * G)^{(n)}\right)}=(m-n) \lambda^{(0)}[f * G],
$$

де $\varrho^{(0)}[f]$ і $\lambda^{(0)}[f]$ відповідно порядок та нижній порядок ряду Діріхле 3 $\sigma_{a}[F]=0$.

У праці отримано аналогічні результати для випадку $\sigma_{a}[F]=+\infty$ i $\sigma_{a}[G] \in$ $(-\infty+\infty)$.

Ключові слова: ряд Діріхле, композиція Адамара, узагальнений порядок, максимальний член. 